\title{
An Issue of Intrusions
}

Intrusive thoughts occur in various forms and have been termed obsessions, worries, flashbacks, negative automatic thoughts etc. Intrusions are both diagnostic features of disorders such as post-traumatic stress, generalized anxiety, obsessive-compulsive disorder, and are also of central theoretical importance in causal models of emotional dysfunction. However, many of these intrusions occur as normal phenomena that are invariant with their clinical counterparts. A main task for theoretical and research efforts is the detailed specification of cognitive-behavioural mechanisms contributing to a pathology of intrusions, and an understanding of their role in the maintenance of certain emotional disorder.

This special issue brings together theoretical, clinical and empirical insights of some of the leading researchers in the field of intrusions. All of the contributors concur that intrusions play a role in the maintenance of certain emotional disorders. Whilst there is diversity in the theoretical models and approaches presented here, there is also a great deal of overlap in themes and concepts. This overlap suggests that generic models capable of explaining a core "dysfunction of intrusions" among a range of disorders may be developed. The overlap stimulates questions concerning the differences between disorders, the differences seem to be less at the level of intrusions themselves and more at the level of the appraisal of intrusions, and the strategies that individuals use to deal with appraised threat.

I would like to thank all of the contributors for submitting their valuable and innovative ideas and research for this special issue of Bebavioural and Cognitive Psychotherapy.

Adrian Wells Associate Editor 